\title{
Erratum to: Fusion with the Cross-Gender Group Predicts Genital Sex Reassignment Surgery
}

William B. Swann Jr. ${ }^{1}$ Ángel Gómez ${ }^{2}$ Alexandra Vázquez ${ }^{2}$ Antonio Guillamón ${ }^{3}$. Santiago Segovia ${ }^{3} \cdot$ Beatriz Carrillo $^{3}$

Published online: 21 March 2015

(c) Springer Science+Business Media New York 2015

\section{Erratum to: Arch Sex Behav}

\section{DOI 10.1007/s10508-014-0470-4}

The surname of this article's last author should be "Carrillo" (not "Carillo").

William B. Swann Jr.

swann@utexas.edu

1 Department of Psychology, College of Liberal Arts, University of Texas at Austin, 1 University Station A8000, Austin, TX 78712, USA

2 Social and Organizational Psychology Department, Universidad Nacional de Educación a Distancia, Madrid, Spain

3 Psychobiology Department, Universidad Nacional de Educación a Distancia, Madrid, Spain 